\title{
A Study to Assess the Awareness of the Biosimilars among Ukrainian Physicians
}

\author{
Ildar Akhmetov, Raheem Farista and Phani Kishore Thimmaraju*
}

Phamax Market Access Solutions, Bangalore, India

\begin{abstract}
Biologics have revolutionized the treatment for severe chronic illness and rare diseases. They represent the fastest growing segment in the pharmaceutical industry. However due to high of innovator biologics, the alternate options such as biosimilars have emerged. The impending patent cliff, rising healthcare costs and increasing awareness of biosimilars among physicians are crucial factors which will drive biosimilar uptake in future. Europe has demonstrated its faith in biosimilar treatment and EMA has approved several biosimilars since 2006. Over the years, Ukraine has been a favourable market for branded generics and its evolving regulatory landscape for biosimilar approval is an indication of its preparedness to embrace biosimilars. The current survey attempts to assess the awareness of the biosimilars among Ukrainian physicians.
\end{abstract}

\section{Introduction}

The last couple of decades were characterized by rapid developments in biotechnology with similar biological medicines frequently replacing conventional methods of treating the long-lasting and chronic diseases. Biosimilars are considered to be amongst the most promising frontiers in pharmacotherapy projected to reach $\$ 25$ billion in global sales by 2020 [1]. The role of biosimilars is also buttressed by rising healthcare costs, impending patent cliff and increasing awareness amid key stakeholders [2]. The attributes of biosimilars propels more and more countries to revise their national policies in order to make the low-cost biologics a reality today. Ukraine is one of those economies that have recently opened the doors to biologics as a new generation treatment of complex diseases.

Unlike the developed economies, the pharmaceutical market of Ukraine is characterized by the significant dominance of branded generics [1]. However, biosimilars are not considered as generics due to their molecular structure, specificity in manufacturing process and requirement for the additional data to prove similarity with the reference product. Ukraine has special marketing authorization provisions for biosimilars and it differs from that of the standard generic drug authorization [3]. Notably, due to cost-effectiveness and proven attributes of the biosimilars in comparison to the innovator product, these products will serve as the accessible options for the physicians.

Today, there are over 300 biological medicines registered in Ukraine, and the number of biosimilar clinical trials is constantly increasing [4]. Teva's filgrastim biosimilar was launched in Ukraine prior to 2015 [5]. Other biosimilars developed by foreign players are trying to enter the nascent segment of the biologics market. In this context, biopharmaceutical companies, like Sandoz (etanercept biosimilar) [6], Boehringer Ingelheim (rituximab biosimilar) [7], Samsung Bioepis (etanercept biosimilar) [8], Biocad (rituximab biosimilar) [9], and Celltrion (trastuzumab biosimilar) [10] have included Ukraine amid their large multinational clinical trials on biosimilars.

\section{Marketing Authorization for Biosimilars in Ukraine}

The Ministry of Health of Ukraine ( $\mathrm{MOH})$ is the regulatory authority that conducts state registration of the medicinal products (including biosimilars) in accordance with the recommendations of 'The State Expert Center'. In Ukraine, $\mathrm{MOH}$ imposes a special requirement to submit additional data when applying for the registration of the biosimilars. Post expiry of the patent protection of the innovator product, the biosimilar applicant has to provide the following materials for registration of the biosimilar:

- Information on pharmaceutical, chemical and biological data, supplemented with bioequivalence and bioavailability data

- Toxicological, pre-clinical and relevant clinical data

- Information pertaining to the identification studies of the biosimilar product

- Clinical data for the biosimilar product for each of the claimed indication in case the reference product is approved for more than one therapeutic indication

\section{Factors Driving Biosimilar Uptake in Ukraine}

In Ukraine, the issue of allowing the biosimilars in the market is regulated by the legislators or government authorities in the assembly. In January 2013, the Ministry of Health of Ukraine $(\mathrm{MOH})$ has amended the Order of $\mathrm{MOH}$ No.426 for the registration of biosimilars. The registration process involves stringent requirements to prove the biosimilarity in terms of safety, efficacy and quality as compared to innovators. Also in July 2013, the Ukraine $\mathrm{MOH}$ has approved the Order No.582 to provide special guidelines on biosimilars. These special guidelines provide the requirements to address the proof of biosimilarity, manufacturing, analytical procedures and bio-activity of the biosimilar drug in comparison with the reference product [11].

In addition, the attractiveness of Ukraine for biosimilar manufacturers is strengthened by the tangible progress in the regulatory space. With an eye on the biosimilar guidelines in

*Corresponding author: Phani Kishore Thimmaraju, Business Analyst at phamax, \#19, KMJ Ascend 1st Cross, 17th C Main 5th Block, Koramangala Bangalore-560095, India, Tel: +91-9916217968; E-mail: phani.kishore@phamax.ch

Received Janaury 06, 2015; Accepted April 17, 2015; Published April 20, 2015

Citation: Akhmetov I, Farista R, Thimmaraju PK (2015) A Study to Assess the Awareness of the Biosimilars among Ukrainian Physicians. J Bioanal Biomed 7: 066-069. doi:10.4172/1948-593X.1000125

Copyright: (c) 2015 Akhmetov I, et al. This is an open-access article distributed under the terms of the Creative Commons Attribution License, which permits unrestricted use, distribution, and reproduction in any medium, provided the original author and source are credited. 
Citation: Akhmetov I, Farista R, Thimmaraju PK (2015) A Study to Assess the Awareness of the Biosimilars among Ukrainian Physicians. J Bioanal Biomed 7: 066-069. doi:10.4172/1948-593X.1000125

development, the Cabinet of Ministries has recently initiated changes in the Law "On Medicines", developing new requirements to biosimilar registration dossiers to elucidate the procedure of proving bioequivalence, safety and efficacy vis-à-vis reference medicines [12]. Furthermore, the focus of the Ukrainian legislators on the national policies to optimize healthcare costs will drive biosimilars uptake in future.

Other factors empowering biotech companies to introduce their biosimilars in Ukraine is the rising healthcare expenditure and the associated need for affordable treatment. Ukraine's healthcare system is a mix of public and private systems, with public expenditure accounting for $57 \%$ of the total healthcare costs, and out-of-pocket funding constituting $~ 93 \%$ of the total private healthcare expenditure [13]. Since the price for novel biologics in Ukraine is comparatively higher than that in the Western Europe, the Ukrainian government expresses its interest in collaborating with biosimilar manufacturers to reduce treatment costs and address the unmet medical needs [14]. Notably, Farmak JSC was the first Ukrainian biotech company to demonstrably prove the cost advantage of biosimilars, introducing its own "replicas" of Lilly France's Humulin (Farmasulin H) at 69\% discount, Sanofi-Aventis' Clexane (Flenox) at 70\% lower price, and Johnson and Johnson's Eprex (Epovitan) at 94\% discount [15].

\section{Current Scenario of Biosimilars in Ukraine}

Despite of the favourable transformation processes for biosimilars, till now the uptake of these medicines among Ukrainian physicians remained limited, due to poor clinical experience and the novelty of a "biosimilar" concept. This has predefined our endeavour to understand the current level of awareness, acceptance and adoption of similar biological medicines among physicians from different therapeutic areas.

\section{Objective of The Study}

The study aims at understanding thelevels of awareness of biosimilars among Ukrainian clinicians in five therapeutic areas (endocrinology, oncology, nephrology, immunology and rheumatology) to outline the development of this segment in Ukraine.

\section{Study design}

1. Study population: Based on the study objective, we have conducted a closed fixed response interview among 82 physicians in five therapeutic areas: endocrinology, oncology, nephrology, immunology and rheumatology (Figure 1).

2. Study Location:

- Berdychiv, Chernyakhiv, Chudniv, Korosten, Malyn,

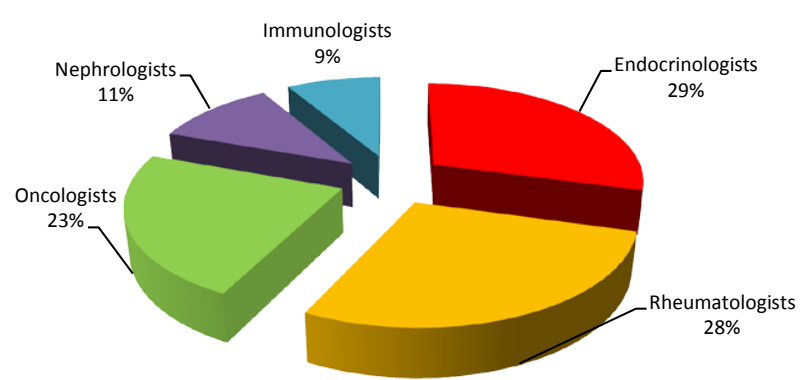

Figure 1: Distribution of the respondents by therapeutic area.
Novograd-Volynsk, Kyiv and Vinnitsa and Zhytomyr.

3. Sampling technique:

- Randomized sampling technique.

- Majority of samples were collected from Zhytomyr, Vinnitsa, Kyiv region due to relatively high demand for biologics and favourable marketing condition.

4. Inclusion criteria: Each respondent had to meet the following selection criteria:

- Over 5 years of work experience in the therapeutic area of focus.

- Previously prescribed biological medicines.

- Working currently in the regions which fall under the geographic scope.

5. Exclusion criteria:

- Interns/house surgeons.

- General physicians who see patients with various ailments which overlap with the specialities listed earlier.

- Visiting physicians who do not belong to the scope of the study location.

6. Questionnaire: A questionnaire contained 8 close-ended questions, including 6 Likert-type items. The duration of the interview was about $\sim 5-7$ min per respondent. The collected data from the respondents has been further modified on a spread sheet and graphically represented through pie charts.

\section{Questionnaire}

1. How many patients do you see per week?
a) $<50$
b) $50-100$
c) $>100$

2. What percent of your patients requires treatment with biological drugs?
a) $<25 \%$
b) $25-50 \%$
c) $50-75 \%$
d) $>75 \%$

3. Of the eligible patients, how many do you prescribe biological drugs for? Please list reasons (if any)?
a) $<25 \%$
b) $25-50 \%$
c) $50-75 \%$
d) $>75 \%$

4. Please, rate your level of awareness on biosimilars using the scale of 1-5 (where $1=$ low and $5=$ high)

5. How did you learn about biosimilars?

a) Patient groups

b) Peer-reviewed journal articles

c) Medical conferences

d) Biotech companies

e) Key ppinion leaders (KOLs)

f) Medical associations

g) Popular press

h) Insurance companies

i) Other 
Citation: Akhmetov I, Farista R, Thimmaraju PK (2015) A Study to Assess the Awareness of the Biosimilars among Ukrainian Physicians. J Bioanal Biomed 7: 066-069. doi:10.4172/1948-593X.1000125

1. If biosimilars are made available, what is your likelihood to prescribe them? (in the scale of $1-5$, where $1=$ low and $5=$ high)

2. What are the main reasons for you to prescribe biosimilars?

a) Trust towards manufacturers of biosimilars

b) Same safety profile as in original biologics

c) Same efficacy in original biologics

d) Cost advantage

e) Propitiousness of reimbursement authorities

f) Propitiousness of patient advocacy groups

g) Other

3. What according to you should be the price for biosimilars in comparison with original drugs?
a) $<10 \%$ lower
b) $10-20 \%$ lower
c) $20-30 \%$ lower
d) $30-40 \%$ lower
e) $40-50 \%$ lower
f) $>50 \%$ lower

\section{Notes}

o Question (1-3): The purpose of the first three questions was to determine the percentage of patients undergoing treatment with biologics, or eligible for this therapy

o Question 4 enabled us to estimate the level of awareness on biosimilars among the studied population

- Question 5 helped us to identify the most prominent sources of information on similar biological medicine

o Question 6 was aimed at measuring the probability to prescribe biosimilars

- Question 7 enabled us to identify the key motives to prescribe similar biological medicines

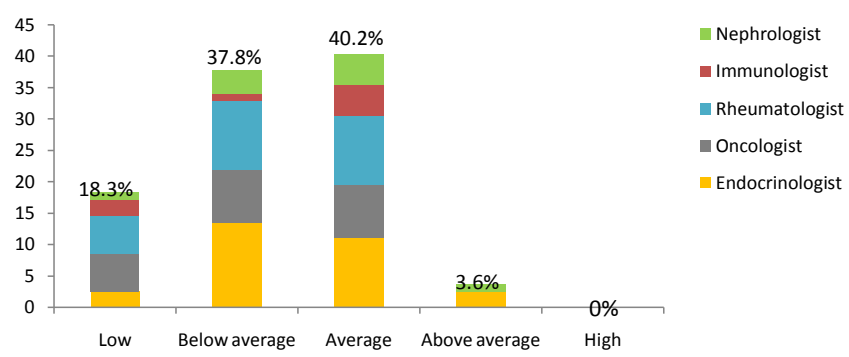

Q: Please rate your level of awareness on biosimilars.

Analysis: The results of the study showed the existing low to medium levels of biosimilar awareness in Ukraine. However, endocrinologists and nephrologists turned out to have higher levels of awareness than other respondents. The availability of biosimilars of filgrastim enabled the nephrologists and endocrinologists understand the significance and their proven efficacy. Lack of approved biosimilars in the areas of immunology and oncology has resulted in poor awareness among physician community in these specialities.

Figure 2: Awareness on biosimilars in Ukraine.

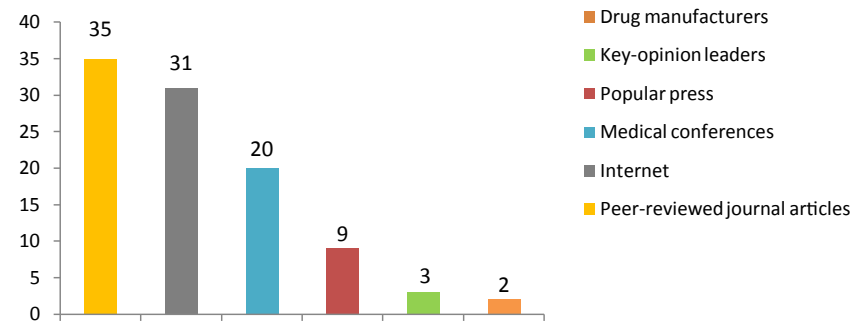

Q: How did you learn about biosimilars?

Analysis: Of all sources of information, peer-viewed journal articles, internet and medical conferences turned out to have the highest impact on physicians awareness and uptake of biosimilars. None of the respondents selected patient advocacy groups, insurance companies or medical associations, which points out on the general underdevelopment of these institutions in Ukraine.

Figure 3: Prominent sources for biosimilar awareness.

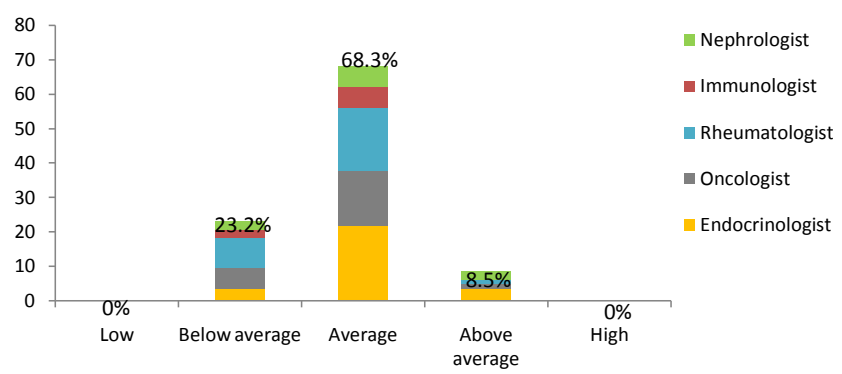

Q: If biosimilars are made available, what is your likelihood to prescribe them? Analysis: At the initial stage, a majority of surveyed physicians are likely to try biosimilars in small batches, and then gradually move to larger groups of patients. Affordability and proven efficacy records will be crucial in driving the prescription patterns of the physicians. Endocrinologists and nephrologists showed greater interest in trying biosimilars among their patients than other practitioners. Moreover, a positive attitude towards biosimilars was observed among respondents with $77 \%$ of surveyed practitioners expressing average and above-average likelihood to prescribe.

Figure 4: Likelihood to prescribe biosimilars.

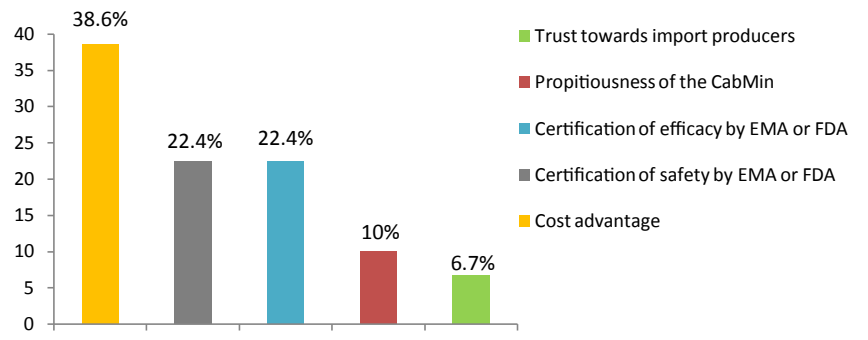

Q: What are the main reasons for you to prescribe biosimilars?

Analysis:As to the intention of prescribing biosimilars, most of physicians preferred cost advantage and certification of safety and efficacy by the EMA or FDA. Very few practitioners selected propitiousness of the Cabinet of Ministers and trust towards import producers (European, American and Japanese biotech companies). We have also observed that the influences of insurance companies, patient advocacy groups and medical associations on physicians decisions to prescribe biosimilars remained frail.

Figure 5: Reasons to prescribe biosimilars.

- Question 8 enabled us to understand an anticipated price difference between biosimilars and reference biologics

\section{Results and Discussions}

The data from the interviews was recorded and analysed on a 


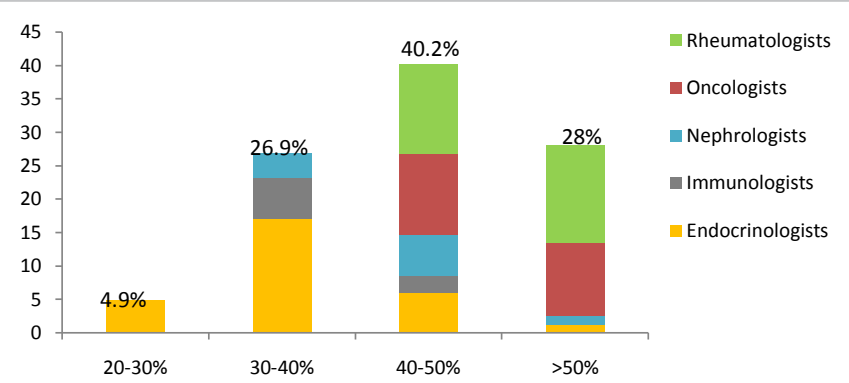

Q: To what extent should the price for biosimilars be lower than the price for original drugs?

Analysis: Regarding the expected price reduction of biosimilars in comparison to innovators, a majority of respondents required $40-50 \%$ lower price for biosimilars than original biologics. Endocrinologists appeared to have the lowest price acceptance margin for biosimilars demanding $20-30 \%$ discount in comparison with the reference medicine, while most of rheumatologists and oncologists anticipated over $50 \%$ price cut. None of respondents selected price options of $<10 \%$ or $10-20 \%$ lower than the innovators.

Figure 6: Price reductions of biosimilars in comparison to innovators.

spread sheet, and graphically represented in the forms of charts. The result is described in this section with analysis of critical questions to meet the study objectives (Figures 2-6).

\section{Conclusion}

Despite of attractiveness of the Ukrainian low-cost biologics market, the study suggests low to medium levels of biosimilar awareness among practitioners in five therapeutic areas. Also, the exposure towards recent developments in biosimilar monoclonal antibodies is also limited which was evident from the analysed responses of immunologists and oncologists. Among those specialists who expressed their willingness to prescribe, are also strong advocates of affordable prices to address the need for low cost biologics. This suggests that biosimilar manufacturers need to spend more effort to educate physicians and address their concern not only pertaining to safety and efficacy but also the cost effectiveness of biosimilars. Unless the above issues are not resolved, it would be difficult to obtain physician's acceptance in Ukraine to prescribe biosimilars.

\section{References}

1. PMR (2012) Changes in reimbursement to bring profits for branded generics in CIS markets between 2012 and 2014

2. IMS Health (2014) Assessing biosimilar uptake and competition in European markets.

3. Gamazin $Y$ (2013) The regulatory landscape for biotech products in Ukraine Health and Medicine.

4. Victorov AP (2011) Preparations biological focus on safe medical use. News of medicine and pharmacy 3 .

5. Adis Insight (2015) Filgrastim biosimilar-Ratiopharm/Teva.

6. GaBI Online (2014) Sandoz advances biosimilars pipeline.

7. Phillippidis A (2013) Biosimilars: 10 Drugs to Watch. GEN news.

8. GaBI Online (2013) Trials to start for biosimilar infliximab and etanercept.

9. PRNewswire (2015) BIOCAD-World Leader in Biosimilar Rituximab Sales

10. GaBI Online (2014) Celltrion starts phase III biosimilar trastuzumab trial.

11. Paliy EV (2014) Biosimilars trying to enter the Ukrainian market. The Ukrainian Journal of Business Law 32-34.

12. Pashkov V (2014) Pharma Market-2014: analysis of the concept of regulatory support. Anteka ua 922.

13. World Bank Database - Ukraine (2014) Public healthcare expenditure, Out-ofpocket healthcare expenditure, Gross per capita income.

14. Johnson K (2012) Access to RA Medications Varies Across Europe. Medscape.

15. Posylkina OV, Litvinov AV (2014) Prospects for the development and the clinical use of biosimilars in Ukraine. Clinical Pharmacy 18: 11-17. 